\title{
TUMORIGENESIS
}

\section{Combined effort}

Probably only a few mutations drive tumour development and progression, but identifying these and defining a therapeutic route through which to exploit them is not easy. Based on their previous findings that cooperating oncogenic mutations lead to synergistic changes in downstream signalling pathways, Hartmut Land and colleagues have shown that a significant number of such synergistic changes are crucial for tumorigenesis.

To identify genes that are regulated synergistically, the authors compared the polysomal mRNA expression profiles of young adult mouse colon cells expressing two cooperative oncogenic mutations, p53-175H and HRAS-V12, with controls. They found 95 annotated genes that exhibited a synergistic change in expression in double mutant cells and these were termed cooperation response genes (CRGs). To assess the relevance of these CRGs to tumorigenesis the authors selected 24 CRGs (based on the extent of the synergy exhibited) and 14 nonCRGs (whose expression changed non-synergistically in response to the oncogenic mutations) and returned their expression back to relatively normal levels using RNA interference or ectopic expression of cDNAs.

Next, they subcutaneously injected the normalized cells into immunocompromised mice and found that normalization of 14 CRGs and only 1 non-CRG significantly reduced tumour formation induced by the cooperative signalling of $\mathrm{p} 53-175 \mathrm{H}$ and HRAS-V12.

So, are the CRGs identified in mice relevant to human cancer? Land and colleagues found that normalization of the homologous CRGs in either DLD1 or HT29 colon cancer cells (which are both p53-null and have oncogenic KRAS or BRAF mutations, respectively) also had a significant inhibitory effect on tumour growth, as determined by xenograft studies in nude mice, indicating that the CRGs are crucial mediators of tumorigenesis.

Therefore, these data demonstrate that synergistic changes in gene expression profiles could be used as a metric to efficiently identify key players that function downstream of oncogenic mutations and might be viable anticancer therapeutic targets.

\section{Gemma K. Alderton}

ORIGINAL RESEARCH PAPER McMurray, H. R. et al. Synergistic response to oncogenic mutations defines gene class critical to cancer phenotype. Nature 25 May 2008 (doi: 10.1038/ nature06973)

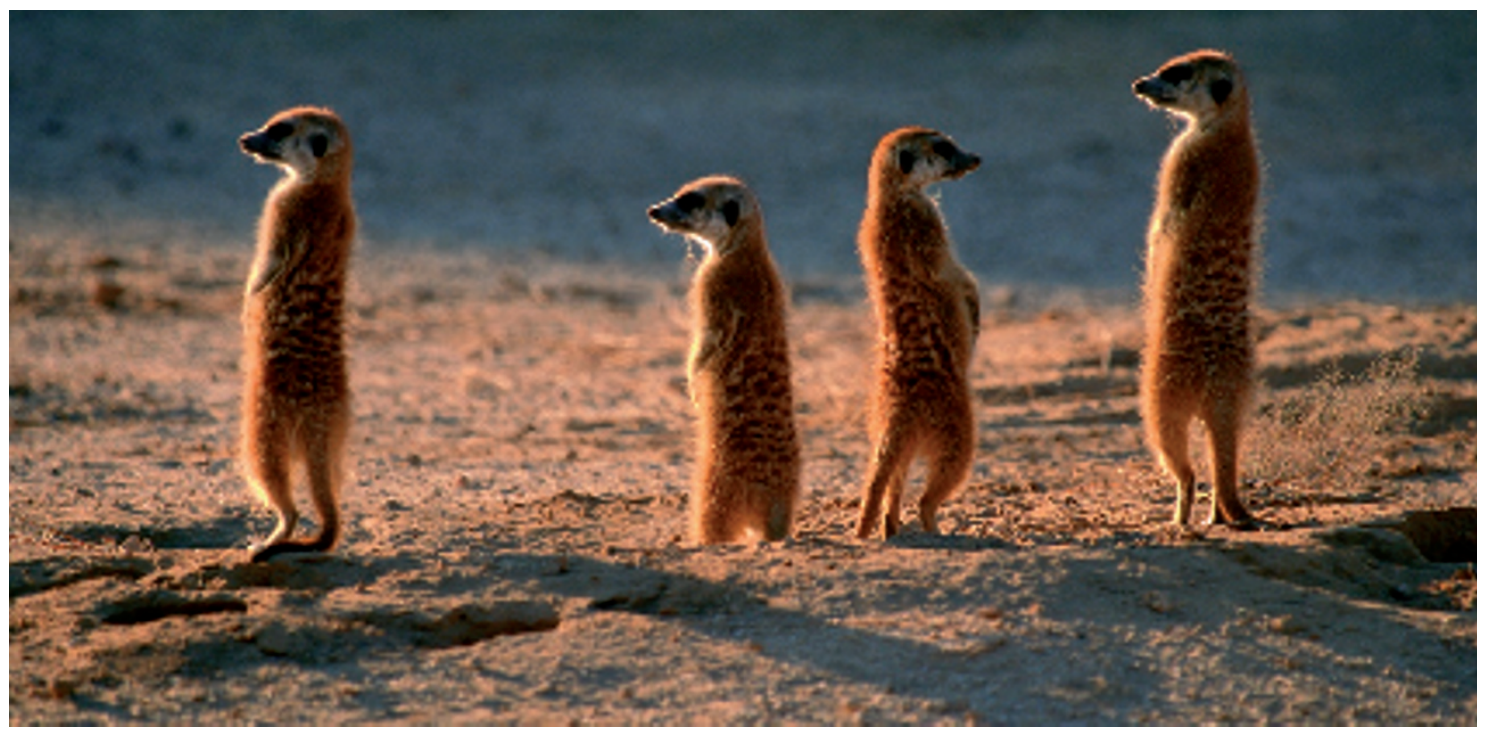

Digital Stock 